\title{
Terahertz reflection imaging using Kirchhoff migration
}

\author{
Timothy D. Dorney and Jon L. Johnson \\ Department of Electrical and Computer Engineering, MS 366, Rice University, Houston, Texas 77251-1892 \\ J. Van Rudd \\ Picometrix, Inc., P.O. Box 130243, Ann Arbor, Michigan 48113-0243
}

Richard G. Baraniuk

Department of Electrical and Computer Engineering, MS 366, Rice University, Houston, Texas 77251-1892

William W. Symes

Department of Computational and Applied Mathematics, MS 134, Rice University, Houston, Texas 77251-1892

Daniel M. Mittleman

Department of Electrical and Computer Engineering, MS 366, Rice University, Houston, Texas 77251-1892

Received May 29, 2001

\begin{abstract}
We describe a new imaging method that uses single-cycle pulses of terahertz $(\mathrm{THz})$ radiation. This technique emulates data-collection and image-processing procedures developed for geophysical prospecting and is made possible by the availability of fiber-coupled $\mathrm{THz}$ receiver antennas. We use a simple migration procedure to solve the inverse problem; this permits us to reconstruct the location and shape of targets. These results demonstrate the feasibility of the $\mathrm{THz}$ system as a test-bed for the exploration of new seismic processing methods involving complex model systems. (C) 2001 Optical Society of America

OCIS codes: $320.7160,100.3010,050.1960$.
\end{abstract}

One important class of imaging problems involves the use of wideband signals. Frequently, these can be posed in the form of an inverse problem in which an impulse is launched at a target and the reflected, transmitted, or diffracted wave is measured at a number of spatial positions. Common examples include ultrawideband radar and pulsed sonar. ${ }^{1-3}$ The latter case is of particular interest because of the complexity of the geophysical imaging problem. Image quality in ground-penetrating sonar can be strongly influenced by factors such as scattering of the propagating acoustic waves. As a result, direct backpropagation of the diffracted wave is often untenable, and a more robust imaging algorithm must be used. ${ }^{3}$ Unfortunately, because the object under study is the Earth, laboratory-scale test-beds for evaluation of these algorithms are frequently unavailable. ${ }^{4}$

In this Letter we demonstrate the feasibility of using terahertz $(\mathrm{THz})$ T-ray imaging as a test-bed for algorithms for solving the inverse problem. We demonstrate the homology between imaging with ultrawideband sonar and with $\mathrm{THz}$ pulses. We simulate seismic data-collection configurations on an optical bench and use migration signal-processing techniques for image formation. ${ }^{5}$ These preliminary results broaden the capabilities of $\mathrm{THz}$ imaging by borrowing a powerful set of tools from a mature imaging community. They also represent the first steps in an attempt to provide a tabletop technique for studying the propagation of few-cycle waves in complex media. Of course, there are important dif- ferences between electromagnetic and acoustic waves, most notably in the issue of longitudinally polarized waves. Nevertheless, there are many similarities as well, and one could hope to uncover very general phenomena through such studies.

Various techniques for imaging with single-cycle $\mathrm{THz}$ pulses were reported recently. ${ }^{6-8}$ For example, Grischkowsky and co-workers explored the use of terahertz time-domain spectroscopy (THz-TDS) for ranging, analogous to ultrawideband pulsed radar measurements. Those experiments emphasized the value of time-domain signal acquisition for elucidating various electromagnetic scattering mechanisms. ${ }^{9,10}$ Johnson et al. reported an interferometric time-of-flight imaging technique that permits resolution well below the Rayleigh limit. ${ }^{11}$ Also, Ruffin et al. demonstrated an image-reconstruction method that involves the backpropagation of secondary wavelets by the time-reversed Kirchoff diffraction integral. ${ }^{12}$ This last example was made possible by a new THz-TDS system in which the gating pulses for both the transmitter and the receiver are delivered via fiber optics. As a result, the antennas can be easily moved without loss of either optical alignment or absolute temporal delay calibration. ${ }^{13}$

The THz-TDS system used in our measurements is identical to the fiber-coupled system described previously. ${ }^{12,13}$ Figure 1(a) shows a representation of the experimental configuration. The emitted radiation reflects off a target and is sensed by an array of receivers. This configuration mimics the 

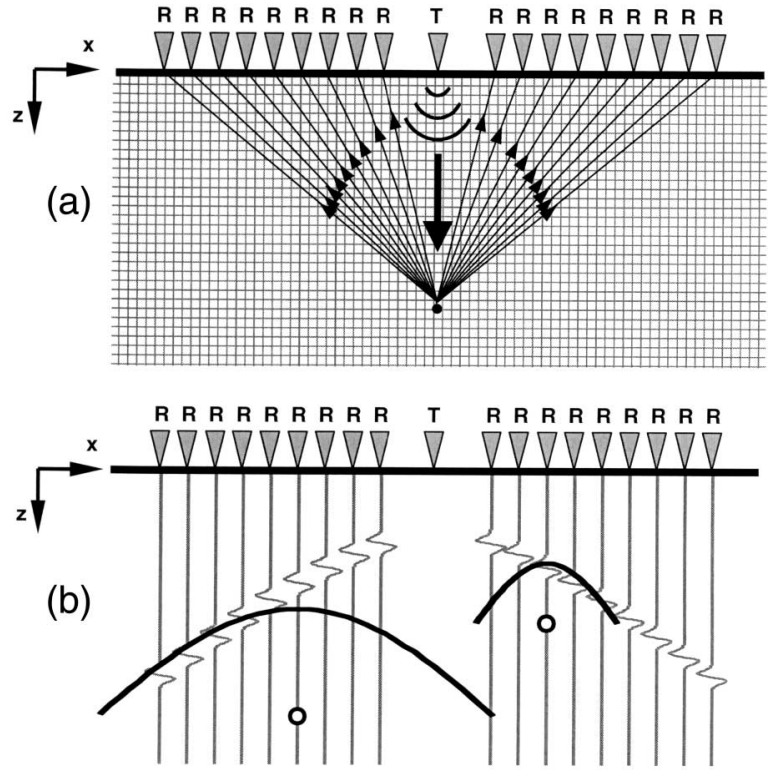

Fig. 1. (a) Schematic of the experimental arrangement emulated by the $\mathrm{THz}$ setup. A single transmitter and multiple, symmetrically placed receivers are arranged to collect a series of reflected waveforms from a point scatterer. The travel time increases hyperbolically with the transmitter-to-receiver separation. (b) Kirchhoff migration reconstructs the location and shape of a reflector by calculating the appropriate hyperbola and summing the values of the recorded traces along that hyperbola for an array of guessed locations. Incorrect locations generate a small summation since their hyperbolas do not pass through many reflected pulses. Two such incorrect guesses and their corresponding hyperbolas are shown.

typical situation in geophysical imaging, in which the transmitter is a device for generating a seismic impulse (e.g., dynamite) and the receivers are an array of geophones. ${ }^{5}$ We approximate this situation by making repeated measurements with a single receiver at many different locations.

It is easy to show that the travel time of a $\mathrm{THz}$ pulse increases hyperbolically with increasing transmitter-to-receiver separation, according to ${ }^{5}$

$$
D(x)=c \tau=\left(x_{0}{ }^{2}+z_{0}^{2}\right)^{1 / 2}+\left[\left(x-x_{0}\right)^{2}+z_{0}^{2}\right]^{1 / 2},
$$

where a point scatterer is located at $\left(x_{0}, z_{0}\right)$ and the receiver is at $(x, 0)$. In general, extended objects also generate hyperbolas, since any surface can be represented as a collection of point scatterers. One unique aspect of this imaging technique is its ability to image extended objects, even those with surfaces tilted with respect to the incoming beam. Conventional $\mathrm{THz}$ reflection measurements have employed a bistatic configuration $^{8,10}$; in this case, if a surface is tilted at a large angle, none of the reflected radiation reaches the receiver. The advantage of multistatic over bistatic imaging has been understood for many years in the radar community. ${ }^{1}$

We illustrate the capabilities of this imaging method by collecting a series of reflected waveforms for a number of different targets. As illustrated in Fig. 1, the targets are located at $x_{0}=0$, the transverse posi- tion of the transmitter. The targets are long metal cylinders of various diameters, located at $z_{0} \approx 90 \mathrm{~mm}$ from the transmitter. We measure the reflected waveforms at each of 152 receiver positions, spaced by $1 \mathrm{~mm}$. Owing to the size of the antenna housing, the smallest transmitter-to-receiver separation is $38 \mathrm{~mm}$. Figure 2 shows the travel time of the measured $\mathrm{THz}$ pulses as a function of transmitter-to-receiver distance for several targets. As expected from Eq. (1), the results are all hyperbolic curves.

One method of forming an image by use of a set of waveforms collected in this fashion is known as Kirchhoff migration. ${ }^{5}$ This method was originally developed for geophysical prospecting but can be readily adapted for $\mathrm{THz}$ imaging. In Fig. 1(b), a simulated series of waveforms is shown vertically, so that distance to the object runs parallel to the temporal axes of the waveforms. The peaks of these waveforms form a hyperbolic curve, as expected from Eq. (1). The migration procedure consists of forming a grid of possible reflector locations and calculating the expected hyperbola for each. Two such possible locations and their corresponding hyperbolas are shown in Fig. 1(b). The numerical value of each pixel in the image is determined by means of summing the values in each waveform along the appropriate hyperbolic curve. For the location corresponding to the actual position of the scatterer, the hyperbola passes through the peaks of every waveform, and a large pixel value results. Because the set of measured waveforms is spaced by a finite amount and covers a finite transmitter-toreceiver range, aliasing effects are inevitable and image artifacts result. We note that migration is quite similar in principle to the method of Ruffin et al. ${ }^{12}$ in the sense that both techniques may be formulated in terms of the propagation of an appropriate Green's function. The distinction is that migration uses only the time-of-flight information and neglects the effect of the decaying amplitude as the transmitter-to-receiver distance increases. ${ }^{14}$ This distinction permits

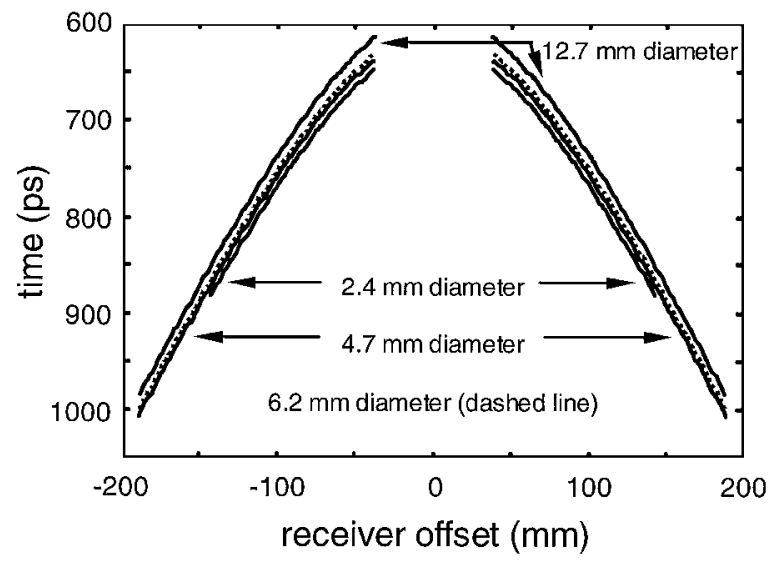

Fig. 2. Measured pulse travel times versus the receiver offset from cylindrical metal targets with diameters as shown. The difference in the curvature of the hyperbolas is very small, yet the difference in the reconstructed images is dramatic (see Fig. 3). The hyperbolas are shifted in time because of the slight variation in transmitter-to-target distances. 


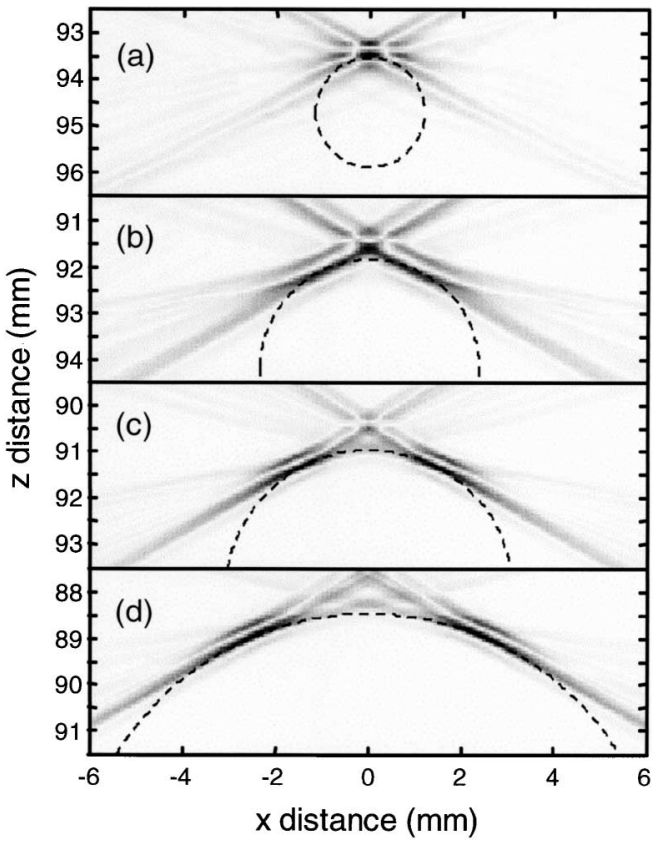

Fig. 3. Kirchhoff migration images from four data sets. The targets are metal cylinders with diameters of (a) $2.4 \mathrm{~mm}$, (b) $4.7 \mathrm{~mm}$, (c) $6.2 \mathrm{~mm}$, and (d) $12.7 \mathrm{~mm}$. The dashed circle and curves represent the outlines of the targets, for comparison with the migration images. In (a), the object is correctly located, but its surface curvature cannot be resolved since its diameter is less than the resolution. In (b) and especially in (c) and (d), both the location of the object and its surface curvature are resolved. The reconstruction of the cylindrical surface is limited by the finite range of receiver offsets.

a simple graphical interpretation such as that shown in Fig. 1(b) and results in a dramatic simplification of the image-formation algorithm.

Figure 3 shows the Kirchhoff migration results for four cylindrical targets. The vertical axes show the distance from the transmitter. For these images, the migration grid spacing (pixel size) is $50 \mu \mathrm{m}$. In all cases, the image places the target at the correct position within our ability to determine it independently. The dashed circle and curves superimposed on these images denote the cross sections of the targets. We note that, although the smallest cylinders are accurately located, they are not well resolved. For the larger cylinders, we begin to resolve the surface curvature. This resolution is determined by the size of the cylinder relative to that of the first Fresnel zone, given by $(c / 4) \sqrt{\tau / \Delta f}$, where $\tau$ is the two-way travel time and $\Delta f$ is the bandwidth of the $\mathrm{THz}$ source. ${ }^{5}$ For our measurements, the horizontal resolution of $\sim 2.9 \mathrm{~mm}$ is somewhat larger than the diameter of the smallest target, which explains our inability to resolve it. Also, the reconstructed curves representing the cylindrical surfaces exhibit a finite thickness. This blurring is a consequence of the finite coherence length of the radiation, which limits the vertical resolution. ${ }^{3}$ In our case, the coherence length is $c / 4 \Delta f \approx 0.19 \mathrm{~mm}$, consistent with the observed surface blurring of 3-4 pixels. Of course, the surface reconstruction is limited in scope because of the finite range of receiver offsets. Finally, we observe a number of image artifacts arising from aliasing effects as mentioned above.

In conclusion, we describe a new image-acquisition procedure that uses a fiber-coupled THz-TDS system. This arrangement emulates the techniques of seismic imaging and borrows data-processing methods from this mature field. The resulting images provide detailed information about the targets, using a very simple and easily implemented algorithmic approach. These results demonstrate the feasibility of the $\mathrm{THz}$ system as a test bed for the exploration of new seismic processing methods involving more complex model systems.

This work was partially supported by the Environmental Protection Agency and the National Science Foundation. T. Dorney's e-mail address is tdorney@ieee.org.

\section{References}

1. R. Sullivan, Microwave Radar: Imaging and Advanced Concepts (Artech House, Norwood, Mass., 2000).

2. A. Rihaczek and S. Hershkowitz, Radar Resolution and Complex-Image Analysis (Artech House, Norwood, Mass., 1996).

3. J. Scales, Theory of Seismic Imaging (Springer-Verlag, Berlin, 1995).

4. J. Ass'ad, R. Tatham, J. McDonald, T. Kusky, and J. Jech, Geophys. Prospect. 41, 323 (1993).

5. M. Dorbin and C. Savit, Introduction to Geophysical Prospecting, 4th ed. (McGraw-Hill, New York, 1988).

6. B. B. Hu and M. Nuss, Opt. Lett. 20, 1716 (1995).

7. D. Mittleman, R. Jacobsen, and M. Nuss, IEEE J. Sel. Top. Quantum Electron. 2, 679 (1996).

8. D. Mittleman, S. Hunsche, L. Boivin, and M. Nuss, Opt. Lett. 22, 904 (1997).

9. R. Cheville, R. McGowan, and D. Grischkowsky, Phys. Rev. Lett. 80, 269 (1998).

10. R. McGowan, R. Cheville, and D. Grischkowsky, IEEE Trans. Microwave Theory Tech. 48, 417 (2000).

11. J. Johnson, T. Dorney, and D. Mittleman, Appl. Phys. Lett. 78, 835 (2001).

12. A. Ruffin, J. Decker, L. Sanchez-Palencia, L. LeHors, J. Whitaker, T. Norris, and J. Rudd, Opt. Lett. 26, 681 (2001).

13. J. Johnson, J. Rudd, and D. Mittleman, Opt. Lett. 25, 1556 (2000).

14. P. Zhao, N. Uren, F. Wenzel, P. Hatherly, and J. McDonald, Geophysics 63, 2072 (1998). 